Corrigendum

\title{
Corrigendum to "Agro-Residues: Surface Treatment and Characterization of Date Palm Tree Fiber as Composite Reinforcement"
}

\author{
Mohamed S. Aly-Hassan, ${ }^{1}$ Elsayed A. Elbadry, ${ }^{2}$ and Hiroyuki Hamada ${ }^{1}$ \\ ${ }^{1}$ Department of Advanced Fibro-Science, Graduate School of Science and Technology, Kyoto Institute of Technology, \\ Matsugasaki, Sakyo-Ku, Kyoto 606-8585, Japan \\ ${ }^{2}$ Mining and Metallurgical Engineering Department, Faculty of Engineering, Assiut University, Assiut 71515, Egypt \\ Correspondence should be addressed to Mohamed S. Aly-Hassan; hassan@kit.ac.jp \\ Received 26 March 2015; Accepted 7 April 2015 \\ Copyright (c) 2015 Mohamed S. Aly-Hassan et al. This is an open access article distributed under the Creative Commons Attribution \\ License, which permits unrestricted use, distribution, and reproduction in any medium, provided the original work is properly cited.
}

Through this corrigendum the authors declare that two coauthors were missing in the paper entitled "Agro-Residues: Surface Treatment and Characterization of Date Palm Tree Fiber as Composite Reinforcement." The correct list of the authors and their affiliations are as shown above. 

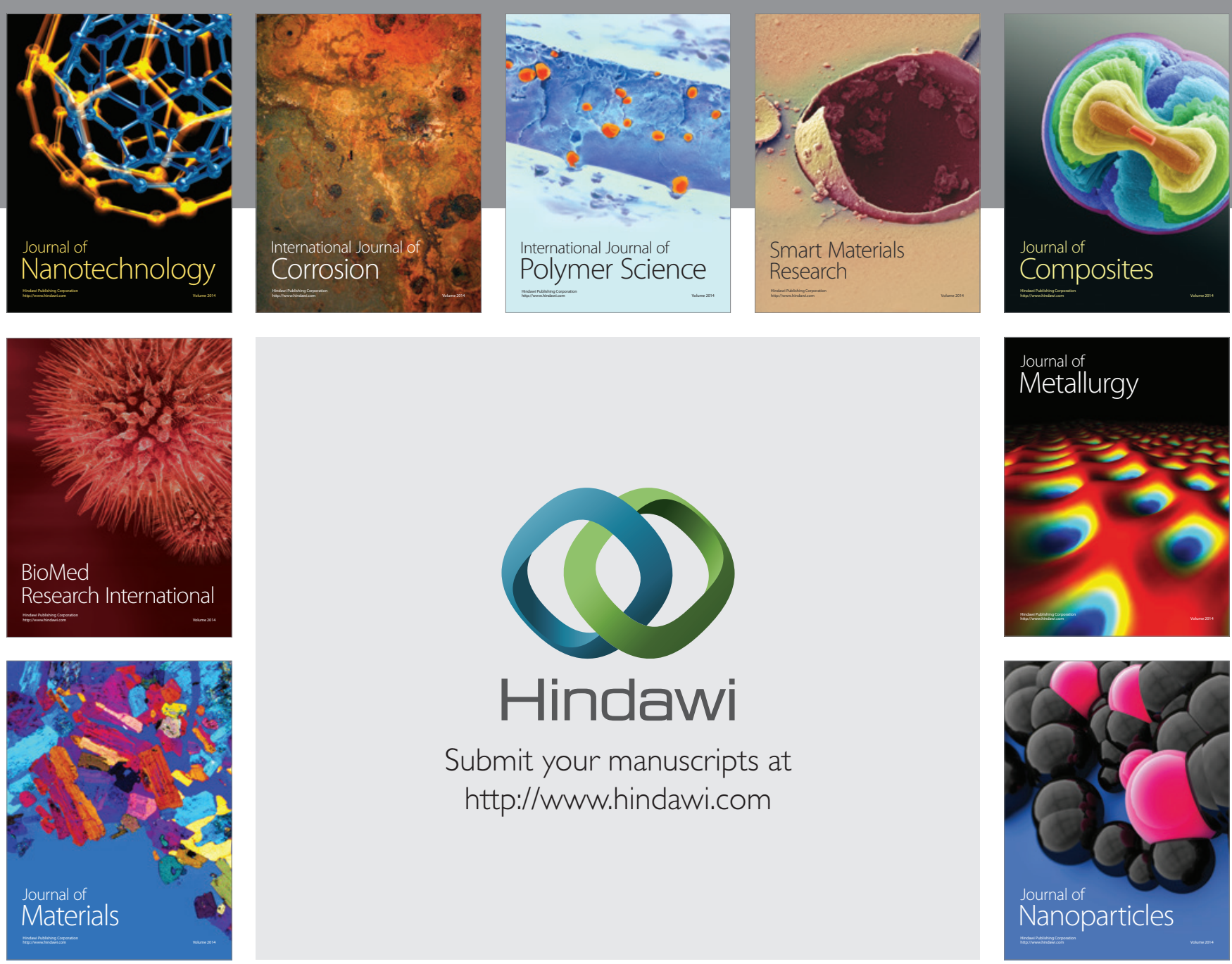

Submit your manuscripts at http://www.hindawi.com
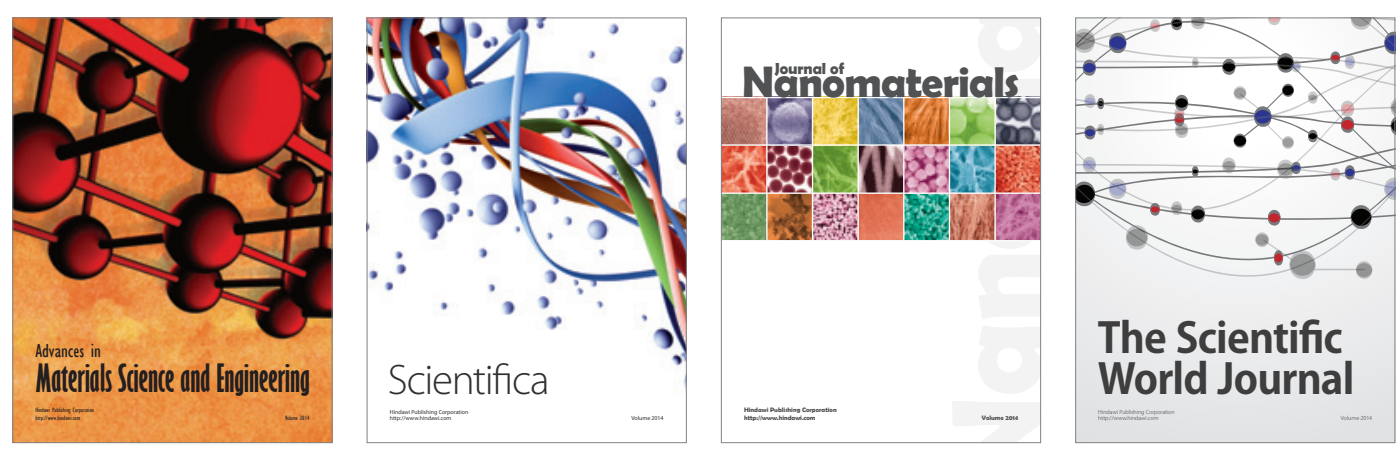

\section{The Scientific World Journal}
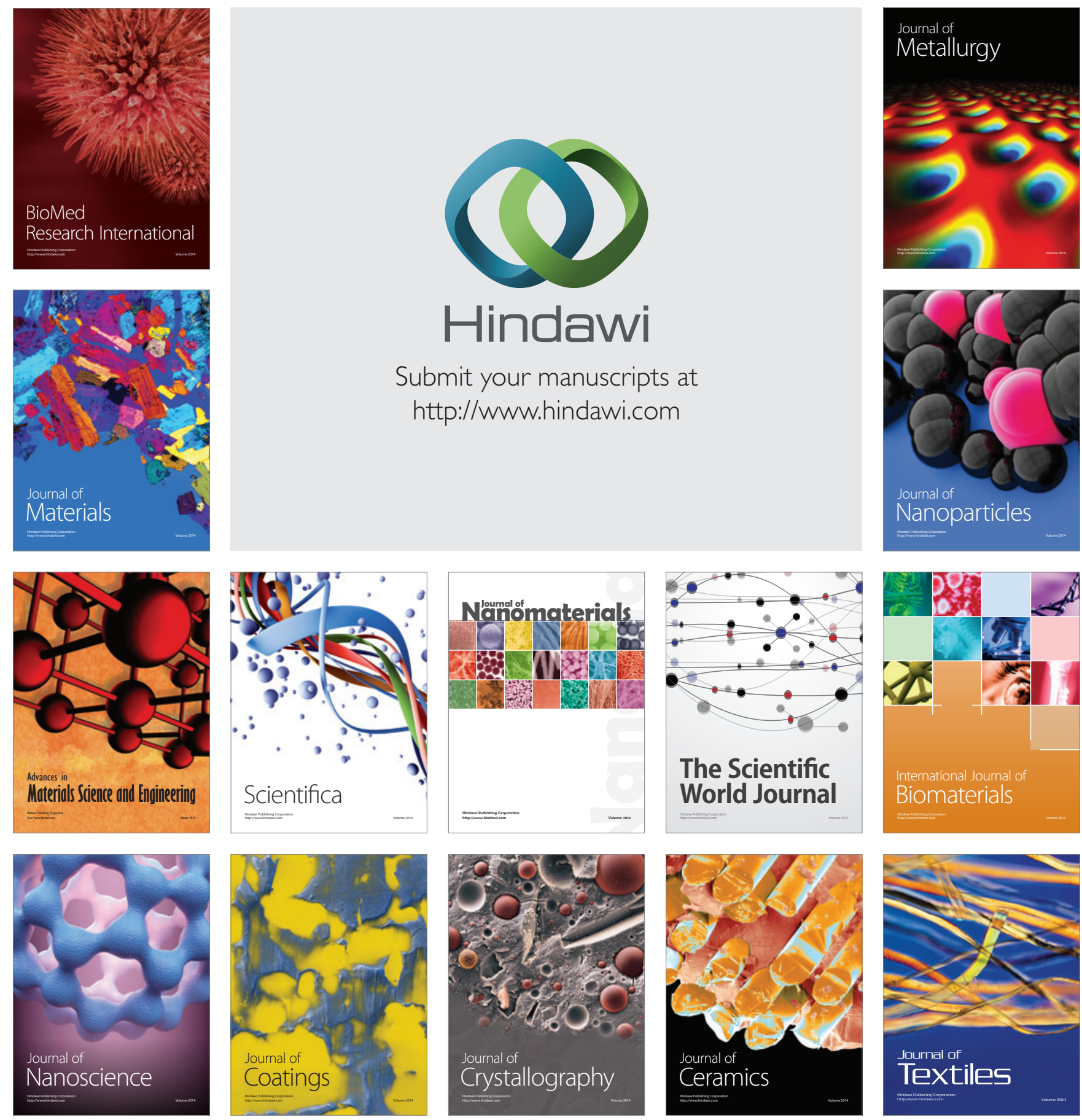